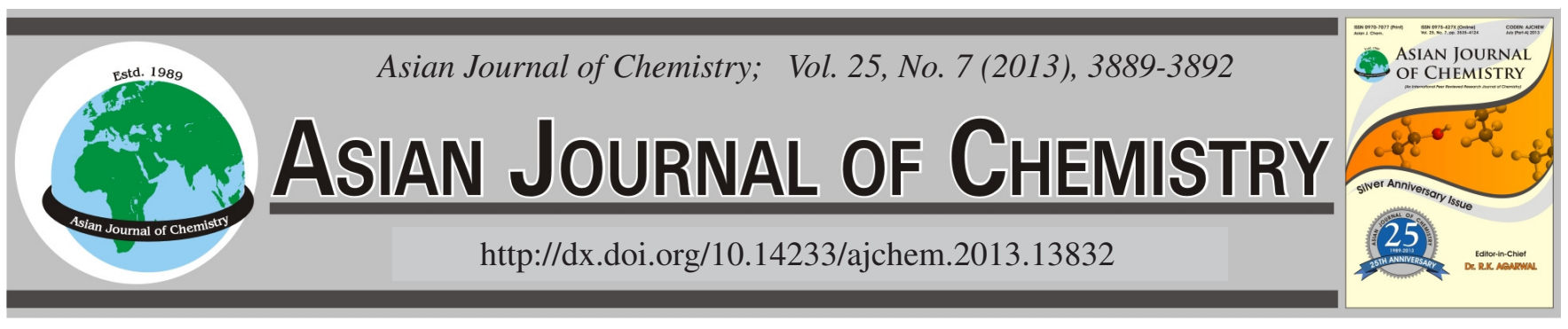

\title{
Pressurized Liquid Extraction and HPLC Analysis for Determination of Polyphenols in Tobacco
}

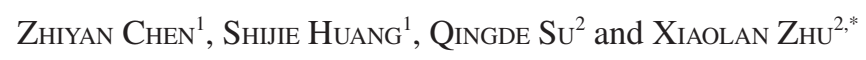

${ }^{1}$ Technical Center, China Tobacco Guangxi Industrial Corporation, Nanning 530001, P.R. China

${ }^{2}$ Research Center of Tobacco and Health, University of Science and Technology of China, Hefei 230052, P.R. China

*Corresponding author: Fax: +86 551 3492065; Tel: +86 551 3492060; E-mail: zx18906@ustc.edu.cn

(Received: 3 April 2012;

Accepted: 14 January 2013)

AJC-12717

\begin{abstract}
A simple and efficient pressurized liquid extraction followed by HPLC analysis method has been investigated for the extraction of polyphenols in tobacco. After pressurized liquid extraction and $\mathrm{C}_{18}$ disposable cartridge solid-phase extraction clean-up, to reduce the co-extraction of interference, polyphenols (chlorogenic acid, esculetin, rutin, scopoletin and quercitrin) were analyzed by HPLC with a diode array detector. When the method was validated, recoveries obtained were varied from 86.9 to $96.4 \%$ and RSDs from 3.4 to $8.2 \%$.

Key Words: Pressurized liquid extraction, Polyphenol, Tobacco, Solid-phase extraction.
\end{abstract}

\section{INTRODUCTION}

Polyphenols in tobacco (Nivotiana tabacum L.) leaves greatly affect tobacco quality and they mainly consist of tannins (chlorogenic acid), coumarins (scopoletin, scopolin) and flavonoids (rutin) ${ }^{1}$. These compounds are precedents of many flavour substances and form a kind of important reaction with amino acids in tobacco ${ }^{2}$. Due to these effects, research on separation and determination of polyphenols in tobacco appears attractive.

LC-MS has been applied in the analysis of these compounds as a suitable technique ${ }^{3}$. However, this hyphenated technique is very expensive and consequently not widely accepted in routine analysis. In routine work, determination of polyphenols with HPLC is the preferred method, owing to its sensitivity, precision and low cost. After an appropriate sample extraction and clean-up procedure, this technique has been used for the determination of polyphenols in fruits ${ }^{4}$, vegetables ${ }^{5}$ and plants ${ }^{6}$.

Pressurized liquid extraction (PLE), often named, accelerated solvent extraction (ASE) has significantly streamlined sample preparation for solid/semi-solid environmental samples ${ }^{7,8}$. It allows reduction of both extraction time and organic solvent consumption and increases sample throughput. Pressurized liquid extraction has been used successfully for the extraction of a wide range of organic compounds in a variety of samples ${ }^{9}$.

Because of the great extracting power of PLE, the extract obtained contains numerous interfering substances, which makes its purification mandatory. The methods of purification most commonly used are solvent-solvent extraction (SSE), gelpermeation chromatography (GPC) ${ }^{10}$ and solid-phase extraction (SPE) with glass columns or commercial cartridges. Solventsolvent extraction consumes large amounts of solvents, disposal of which environmentally hazardous. Gel-permeation chromatography is costly and is always combined with other cleanup procedures ${ }^{11}$. Compared with such clean-up techniques, SPE has advantages of, for example, simplicity, speed, efficient use of solvents and use of different types of adsorbent to meet the needs of the analysis.

In our previous work ${ }^{12}$, a dynamic ultrasound-assisted extraction as sample preparation method for analysis of polyphenols had been developed. The objective of this study was to establish and validate a method based on PLE with SPE purification for analysis of polyphenols in tobacco. The optimized HPLC-DAD conditions and three wavelength channel detections are discussed. Using the proposed method, five polyphenols (chlorogenic acid, esculetin, scopoletin, rutin and quercitrin) of dry ground tobaccos are directly identified and quantified.

\section{EXPERIMENTAL}

Standards of chlorogenic acid, esculetin, scopoletin, rutin, quercitrin and kaempferol were purchased from SigmaAldrich (Sigma-Aldrich Inc., Germany). Acetonitrile and methanol (Tedia Inc. OH, USA), used for the mobile phase or the extraction solvent, were all of HPLC-grade. 
Celite (30-80 mesh) and diatomaceous earth (200-260 mesh) were obtained from Shanghai Refine Chemical Factory (SRCF, Shanghai, China) and of analytical-grade. Ultra-pure water was obtained from a water purification system in our own Lab. Analytical-reagent grade materials were used unless otherwise indicated. The $\mathrm{C}_{18} \mathrm{SPE}$ cartridges $(3 \mathrm{~mL} / 500 \mathrm{mg}$ ) investigated for the clean-up step were purchased from Dikma Ltd (Dikma Co., Beijing, China).

All the adsorbents were directly filled into the filtration or clean-up cartridge without deactivation. All standard solutions were prepared by dissolving each of the six polyphenols standards in anhydrous methanol and stored at $-20^{\circ} \mathrm{C}$ in the dark, where they could be stable for at least 2 months. Solutions of single standards and the mixture of all were prepared weekly by appropriate dilution of the stock solution with the acetonitrile and $0.2 \%$ trifluoroacetic acid aqueous solution $(1: 2, \mathrm{v} / \mathrm{v})$. Mobile phases were filtered through $0.45 \mu \mathrm{m}$ Millipore membranes and degassed before HPLC analysis.

Pressurized liquid extraction and cleanup procedure of polyphenols: Automated PLE was performed with an ASE 300 system with $34 \mathrm{~mL}$ stainless steel extraction cell (Dionex, Sunnyvale, USA). Tobacco sample (5.000 g) was mixed with $10.00 \mathrm{~g}$ diatomaceous earth and the mixture was added directly to the extraction cell containing cellulose extraction filters to prevent fine powder breakthrough into the collection bottle. Extraction was performed under the following conditions: extraction solvent anhydrous methanol (containing $0.5 \%$ ascorbic acid), temperature $40^{\circ} \mathrm{C}$, pressure $1500 \mathrm{psi}$, static time $5 \mathrm{~min}$, heat-up time $5 \mathrm{~min}$, flush volume $60 \%$, purge $\mathrm{N}_{2}$ for $1 \mathrm{~min}$ and number of cycles 2 . Finally, the extracts were concentrated under reduced pressure and employed to $5 \mathrm{~mL}$ for the clean-up step.

A $0.5 \mathrm{~mL}$ of the extract solution was loaded onto the Waters $\mathrm{C}_{18}$ disposable cartridge for clean-up. After the washing and elution procedure, the elute was dried and reconstituted in $1.0 \mathrm{~mL}$ with mobile phase, filtered and $10 \mu \mathrm{L}$ was injected into the HPLC system for analysis.

Ultrasound-assisted extraction (UAE) of polyphenols: Dry ground tobacco (5 g, 60-80 mesh) and anhydrous methanol (50 mL, containing $0.5 \%$ ascorbic acid) were placed in a $150 \mathrm{~mL}$-volumetric flask. The sealed flask was immediately transferred to the ultrasonic apparatus and extracted at room temperature for $1 \mathrm{~h}$. Following sonication, the solution was filtered through glass wool and the residue was re-extracted with $50 \mathrm{~mL}$ anhydrous methanol (containing $0.5 \%$ ascorbic acid). Finally, the extract was combined and concentrated under reduced pressure and employed to $5 \mathrm{~mL}$ for the clean-up step.

HPLC analysis: Chromatographic analyses were performed on an Agilent 1100 system, equipped with a binary pump, an autosampler and a DAD, connected to Agilent ChemStation software. A RP Waters $\mathrm{C}_{18}$ column $(250 \mathrm{~mm} \times 4.6 \mathrm{~mm} \mathrm{ID,} 5$ $\mu \mathrm{m}$ particle size, Waters) was used for separation $\left(\mathrm{T}=35^{\circ} \mathrm{C}\right)$. Solvents that constituted the mobile phase were A (acetonitrile) and $\mathrm{B}(0.03 \%$ trifluoroacetic acid aqueous solution, v/v). The elution conditions applied were as follows: 0-2 min $86 \% \mathrm{~B}$ isocratic; 2-20 min, linear gradient of 86-65\% B; $27 \mathrm{~min}, 20$ $\% \mathrm{~B} ; 33 \mathrm{~min}, 20 \% \mathrm{~B}$ and finally, washing and reconditioning of the column with initial phases. The flow rate used was 1.0 $\mathrm{mL} / \mathrm{min}$. The spectra of five polyphenols were obtained through the Agilent 3D ChemStation software scanning at the range of 190-400 $\mathrm{nm}$ with acetonitrile and $0.03 \%$ trifluoroacetic acid aqueous solution as mobile phases, where the maximum absorption wavelengths for chlorogenic acid, esculetin, rutin, scopoletin and quercitrin were observed at 346 and 330 $\mathrm{nm}$. Thereby, double-beam detection was employed in analysis of these compounds in tobacco.

\section{RESULTS AND DISCUSSION}

Optimization of pressurized liquid extraction conditions: During PLE extraction of polyphenols, extract solvent, temperature and heating time are prime attributes affecting extraction recovery. To optimize these conditions, a three-level orthogonal array design (OAD) was employed. The extract solvent, temperature and heating time were the optimized variables with the constant sample amount (5.000 g).

Aqueous methanol is often selected as extraction solvent for the polyphenols because of its property of co-dissolution with the polyphenols ${ }^{13}$. It was also reported that a higher content of methanol in the aqueous solution would favour the extraction of large molecule polyphenolic compounds ${ }^{14}$. In order to protect the target polyphenols from decomposition and oxidation, we selected anhydrous methanol containing $0-1.0 \%$ ascorbic acid as extraction solvent ${ }^{15}$.

The results of extraction of polyphenols are presented in Table-1. After the OAD procedure had been conducted, a graph with the sum of the amount of polyphenols with the same extract temperature or heating time level was drawn to examine the key variable. From the graph (Fig. 1), it may be seen that extract solvent is the important variable influencing the sum of amount of polyphenols. The sum of the amount of polyphenols obtained with methanol containing $0.5 \%$ ascorbic acid (46.215 $\mathrm{mg} / \mathrm{g}$ ) was much higher than those obtained with methanol $(33.211 \mathrm{mg} / \mathrm{g})$ or methanol containing $1 \%$ ascorbic acid (42.145 $\mathrm{mg} / \mathrm{g})$. These may be because ascorbic acid could prevent polyphenols from oxidation and decomposition. The temperature as well as extract time in the range studied played a less important role. Therefore, the optimum extract solvent, temperature and extract time for extract tobacco samples were methanol containing $0.5 \%$ ascorbic acid, $40{ }^{\circ} \mathrm{C}$ and $10 \mathrm{~min}$, respectively.

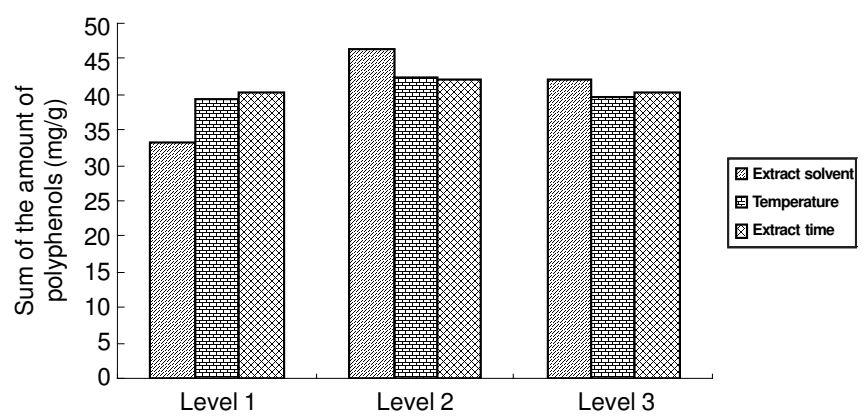

Fig. 1. Effects of extract solvent, temperature and heating time on the amount of polyphenols extraction

Optimization of clean-up: Using anhydrous methanol as a polar extraction solvent, the polar compounds could be coextracted, which can interfere with the HPLC separation of polyphenol. A cleanup step is necessary in the analytical process, of which solid-phase extraction is a common method ${ }^{16}$. Considering the capacity of $\mathrm{C}_{18}$ disposable cartridge and the 
TABLE-1

RESULTS OF PLE OF POLYPHENOLS $\left(\mathrm{L}_{9}(3)^{4}\right.$ ORTHODOXY TABLE)

\begin{tabular}{clcccc}
\hline No. & Extract solvent & Temperature $\left({ }^{\circ} \mathrm{C}\right)$ & Extract time $(\mathrm{min})$ & Amount of PPs ${ }^{\mathrm{a}}(\mathrm{mg} / \mathrm{g})$ & $\mathrm{RSD}^{\mathrm{b}}(\%)$ \\
\hline 1 & Methanol & Room temperature & 5 & 10.564 & 11.641 \\
2 & Methanol & 40 & 10 & 11.006 & 3.2 \\
3 & Methanol & 60 & 15 & 15.632 & 3.9 \\
4 & Methanol (0.5\% AA $)$ & Room temperature & 10 & 15.897 & 2.4 \\
5 & Methanol (0.5\% AA) & 40 & 15 & 14.686 & 1.9 \\
6 & Methanol (0.5\% AA) & 60 & 5 & 13.256 & 2.8 \\
7 & Methanol (1.0\% AA) & Room temperature & 15 & 14.892 & 3.4 \\
8 & Methanol (1.0\% AA) & 40 & 5 & 13.997 & 4.1 \\
9 & Methanol (1.0\% AA) & 60 & 10 & 3.7 \\
\hline
\end{tabular}

${ }^{a}$ Amount of PPs is sum of the amount of five polyphenols. ${ }^{b} \mathrm{RSD}=$ Relative standard deviation, $\mathrm{n}=3 .{ }^{\mathrm{c}} \mathrm{AA}$ is ascorbic acid.

complex property of tobacco extract, the breakthrough of the cartridge was investigated with the spiked and nonspiked samples.

In the work of Oliveira et al., the breakthrough of $\mathrm{C}_{18}$ cartridge was not investigated ${ }^{17}$. To investigate the breakthrough of $\mathrm{C}_{18}$ cartridge in our experiment, $5 \mathrm{~g}$ tobacco samples were extracted and the extract was concentrated to $5 \mathrm{~mL}$. Then, $0.25,0.5,1.0$ and $2.0 \mathrm{~mL}$ volumes of the concentration extract were loaded onto the clean-up cartridge. The results showed the clean-up recoveries were lower than $98 \%$ when the volume was greater than $0.5 \mathrm{~mL}$ and continuously decreased with increasing of volume. This is probably because of insufficient column capacity of $\mathrm{C}_{18}$ cartridge or leaching by extract itself. Chromatogram is shown in Fig. 2. This clean-up step was also helpful for elimination of lipid compounds probably extracted by methanol to prevent the contamination of the analytical column. Then the $\mathrm{C}_{18}$ cartridge was washed with $3 \mathrm{~mL}$ of 0.01 $\mathrm{mol} / \mathrm{L}$ hydrochloric acid solution and eluted with $20 \mathrm{~mL}$ methanol. The elutes were evaporated to a droplet by rotary evaporation and dried by means of a gentle nitrogen stream. The final solution was reconstituted in $1 \mathrm{~mL}$ with mobile phase, filtered and $10 \mu \mathrm{L}$ was injected into the HPLC system for analysis.

HPLC separation and identification of the six polyphenols: Polyphenols are difficult to ionize in acidic conditions; acetonitrile was reported to separate polyphenols well ${ }^{18}$. Therefore, $0.2 \%$ aqueous solution of trifluoroacetic acid and acetonitrile were selected as mobile phases in this experiment. It should be noted that if the content of trifluoroacetic acid were higher than $0.5 \%$, the $\mathrm{pH}$ of the solution would be lower than 2 , which would damage the separation systems under the proposed conditions. In order to enhance the detection limit, a multichannel data acquisition technique was adopted; thus threewavelength simultaneous detection was employed in this HPLC analysis. DAD-UV at $346 \mathrm{~nm}$ was used for determination of esculetin, scopoletin, rutin and quercitrin and at $330 \mathrm{~nm}$ for chlorogenic acid (Fig. 2).

The identification of the five polyphenols was based on the following aspects: (1) retention times; (2) comparison of the UV spectra of the target compounds with that of the corresponding standard solutions under the same condition; (3) standard addition method. Quantitation of the compounds was based on the relation between peak areas and concentrations utilizing the external standard method. The calibration curves covered concentrations of the six polyphenols from 0.5 to 500 $\mu \mathrm{g} / \mathrm{mL}$. The correlation coefficients for the five polyphenols were all higher than 0.99 .

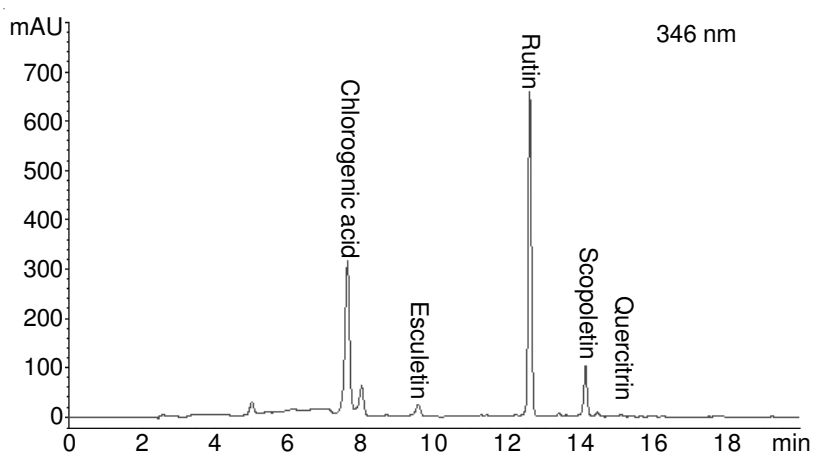

(a)

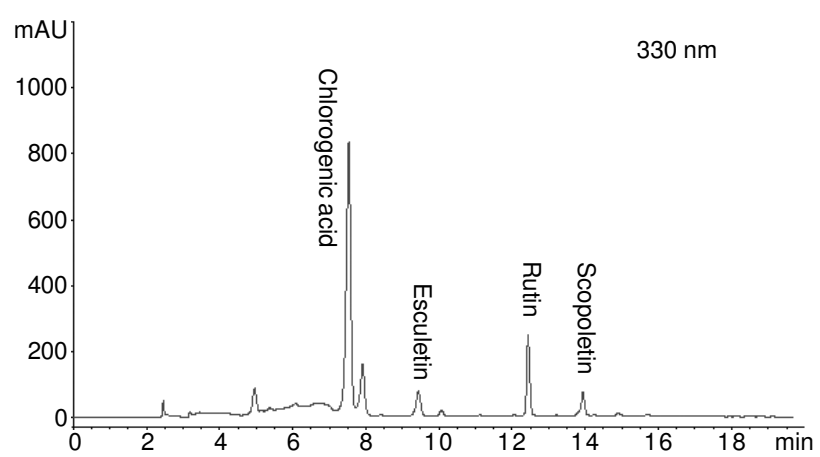

(b)

Fig. 2. Chromatogram at $346 \mathrm{~nm}$ for tobacco sample cleaned up with $\mathrm{C}_{18}$ cartridge (double channel detection with (a) $346 \mathrm{~nm}$ and (b) 330 $\mathrm{nm})$

Validation of the method: To evaluate the validation of the new analytical method, accuracy and precision were considered. One flue-cured sample (Yunnan, China) was used. The spiked samples were prepared by adding mixture polyphenols standard solution to suitable portions of dry ground tobacco. The spiked tobacco was left at room temperature for $2 \mathrm{~h}$ before extraction, in order for polyphenols to be adsorbed on the tobacco and correspond more closely to the field-treated tobaccos. The samples were then extracted and determined as described in preceding procedure. Each level was repeated six times for calculation of recovery and RSD. Recoveries of the five polyphenols are 88.9-96.4\% and the RSD of repeatability are 3.4-6.1\% (Table-2).

Comparison of methods: Some of the properties of PLE are compared to UAE and supercritical fluid extraction (SFE), as listed in Table-3. In PLE, the time needed to extract polyphenols quantitatively from tobacco samples is $10 \mathrm{~min}$. This is much faster than $\mathrm{UAE}$ and $\mathrm{SFE}^{19}$. Solvent consumption was 
TABLE-2

RECOVERIES AND RSDS OF FIVE POLYPHENOLS BY PLE

\begin{tabular}{|c|c|c|c|c|c|c|c|}
\hline \multirow{2}{*}{ Analytes } & \multicolumn{2}{|c|}{ Contents $(\mu \mathrm{g} / \mathrm{mL})$} & \multirow{2}{*}{$\begin{array}{l}\text { Added level } \\
(\mu \mathrm{g} / \mathrm{mL})\end{array}$} & \multicolumn{2}{|c|}{ Recovery (\%) } & \multicolumn{2}{|c|}{$\operatorname{RSD}(n=6)(\%)$} \\
\hline & PLE & UAE & & PLE & UAE & PLE & UAE \\
\hline \multirow{3}{*}{ Chlorogenic acid } & \multirow{3}{*}{3561} & \multirow{3}{*}{3298} & 100 & 91.2 & 88.3 & 4.1 & 5.2 \\
\hline & & & 300 & 92.4 & 93.5 & 3.9 & 6.6 \\
\hline & & & 600 & 90.5 & 91.0 & 4.6 & 5.2 \\
\hline \multirow{3}{*}{ Esculetin } & \multirow{3}{*}{32.2} & \multirow{3}{*}{28.6} & 10 & 95.3 & 93.5 & 5.6 & 5.6 \\
\hline & & & 30 & 94.8 & 91.4 & 4.5 & 7.5 \\
\hline & & & 60 & 96.1 & 94.3 & 6.1 & 6.4 \\
\hline \multirow{3}{*}{ Scopoletin } & \multirow{3}{*}{87.6} & \multirow{3}{*}{85.6} & 20 & 93.8 & 90.7 & 4.6 & 4.9 \\
\hline & & & 60 & 93.4 & 93.6 & 3.8 & 4.8 \\
\hline & & & 120 & 96.4 & 94.4 & 3.4 & 4.4 \\
\hline \multirow{3}{*}{ Rutin } & \multirow{3}{*}{2459} & \multirow{3}{*}{2367} & 100 & 88.9 & 91.6 & 4.6 & 5.2 \\
\hline & & & 300 & 93.6 & 92.0 & 5.1 & 9.4 \\
\hline & & & 600 & 92.0 & 89.6 & 4.7 & 5.7 \\
\hline \multirow{2}{*}{ Quercitrin } & \multirow{2}{*}{18.4} & \multirow{2}{*}{17.3} & 10 & 90.5 & 86.1 & 4.4 & 5.4 \\
\hline & & & 60 & 91.2 & 89.6 & 5.2 & 6.2 \\
\hline
\end{tabular}

\begin{tabular}{lccc} 
& \multicolumn{3}{c}{ TABLE-3 } \\
& PROPERTIES OF PLE, COMPARED TO UAE, SFE \\
\hline \multicolumn{1}{c}{ Properties } & PLE & UAE & SFE \\
\hline Sample consumption $(\mathrm{g})$ & 5 & 5 & 280 \\
Extraction time (min) & 10 & Room temperature & 180 \\
Extraction temperature $\left({ }^{\circ} \mathrm{C}\right)$ & 40 & Over-laborious & 83 \\
Amount of manpower & Labour-saving & 80 & Over-laborious \\
Recoveries $(\%)$ & 89 & & 80 \\
\hline a See Andrich et al. $^{24}$ & &
\end{tabular}

also greatly reduced. UAE was a conventional extraction technique for many compounds ${ }^{20}$, ultrasonic energy was employed to accelerate the extraction process and multiple extraction steps we re used. From Table-2, it could be seen that slightly greater recoveries were obtained with PLE than UAE for the five target polyphenols. This is because of the great extracting power of PLE which promoted desorption of analytes from the solid matrices. Theoretically, the analytes could be "exhaustively" extracted. Except for higher recoveries, organic solvent and time consumption were significantly reduced. SFE employ higher temperatures for extraction and a low recovery was gained with SFE. This is probably because some of the polyphenols were decomposed during extraction.

\section{Conclusion}

In summary, a rapid and reliable pressurized liquid extraction followed by HPLC-DAD separation and detection method was developed and applied to separate, identify and quantify the five polyphenols by three-channel detection at 346 and $330 \mathrm{~nm}$.

\section{ACKNOWLEDGEMENTS}

This work was supported by the Scientific Foundation of Guangxi Tobacco Commercial Corporation (No. 20090056 ). The authors also thank their colleagues for their valuable technical assistance.
3. Y. Wang, F. Catana, Y. Yang, R. Roderick and R.B. van Breemen, J. Agric. Food Chem., 50, 431 (2002).

4. H. Sakakibara, Y. Honda, S. Nakagawa, H. Ashida and K. Kanazawa, J. Agric. Food Chem., 51, 571 (2003).

5. M. Careri, C. Corradini, L. Elviri, I. Nicoletti and I. Zagnoni, J. Agric. Food Chem., 51, 5226 (2003).

6. Y.T. Wu, W.Y. Huang, T.C. Lin and S.J. Sheu, J. Sep. Sci., 26, 1629 (2003).

7. Y. Jhong and W. Ding, J. Chin. Chem. Soc., 55, 335 (2008).

8. Z. Zhang, M. Shan Mugan and S.M. Rhind, Chromatographia, 72, 535 (2010).

9. J.J. Wu, Y.L. Mark, M.B.Murphy, J.C. Lam, W.H. Chan, M. Wang, L.L. Chan and P.K. Lam, Anal. Bioanal. Chem., 400, 3165 (2011).

10. M.A. Presta, D.I.S. Kolberg, C. Wickert, I.R. Pizzutti, M.B. Adaime and R. Zanella, Chromatographia, 69, 237 (2009).

11. D.G. Hayward and J.W. Wong, Anal. Chem., 81, 5716 (2009).

12. X.G. Gu, J.B. Cai, X.L. Zhu and Q.D. Su, J. Sep. Sci., 28, 2477 (2005).

13. Y.G. Zuo, H. Chen and Y.W. Deng, Talanta, 57, 307 (2002).

14. L.H. Yao, Y.M. Jiang, N. Datta, R. Singanusong, X. Liu, J. Duan, K. Raymont, A. Lisle and Y. Xu, Food Chem., 84, 253 (2004).

15. F. Chinnici, A. Gaiani, N. Natali, C. Riponi and S. Galassi, J. Agric. Food Chem., 52, 3 (2004).

16. Y. Zhang, J. Yang, R. Shi, Q. Su, Y. Gao and X. Zhu, Chromatographia, 73, 385 (2011).

17. E.C. Oliveira, M. do, C.R. Peralba and E.B. Caram, J. Sep. Sci., 25, 356 (2002).

18. B. Meier, R.J. Tiitto, J. Tahvanainen and O. Sticher, J. Chromatogr., 422, 175 (1998).

19. G. Andrich, S. Balzini, A. Zinnai, V. De Vitis, S. Silvestri, F. Venturi and R. Fiorentini, Eur. J. Lipid Sci. Technol., 103, 151 (2001).

20. V. Lopez-Avila, R. Young and N. Teplitsky, J. AOAC Int., 79, 142 (1996).

\section{REFERENCES}

1. A.G. Kallianos, Recent Adv. Tob. Sci., 20, 61 (1976).

2. T. C. Tso, Production, Physiology and Biochemistry of Tobacco Plant, Beltsville, Maryland, Ch. 25, pp. 533-568. 